\title{
Screening Circulating Tumor Cells as a Non-invasive Cancer Test in 1,585 Asymptomatic Adults (ICELLATE1)
}

\author{
Juan Castro ${ }^{1}$, Luis Sanchez ${ }^{2}$, Pierino H Alvarez Bedoya ${ }^{2}$, María Teresa Nuñez ${ }^{3}$, Ming Lu ${ }^{4}$, Tomas Castro ${ }^{4}$, Hamid R Sharifi ${ }^{4,5}$ and Christer Ericsson ${ }^{4,5^{*}}$ \\ ${ }^{1}$ Department of Oncology-Pathology, Karolinska Univ Hospital, Sweden \\ ${ }^{2}$ Hospital Nacional Arzobispo Loayza, Av Alfonso Ugarte 848, Cl Javier Heraud 320, Urb Covima, La Molina, Lima, Peru \\ ${ }^{3}$ Department of Pathology, Instituto Nacional de Enfermedades Neoplasicas (INEN), El Condado St. 212 La Molina, Lima 12, Peru \\ ${ }^{4}$ iCellate Medical AB, KI Science Park, Industrivägen 1, Sweden \\ ${ }^{5}$ Department of Microbiology, Tumor and Cell Biology (MTC), Biomedicum 8C, Karolinska Institutet, Sweden
}

*Corresponding author: Christer Ericsson, Department of Microbiology, Tumor and Cell Biology (MTC), Biomedicum 8C, Karolinska Institutet, Sweden; E-mail: christer.ericsson@ki.se

Received date: July 18, 2018; Accepted date: September 03, 2018; Published date: September 07, 2018

Copyright: (c) 2018 Castro J, et al. This is an open-access article distributed under the terms of the Creative Commons Attribution License, which permits unrestricted use, distribution, and reproduction in any medium, provided the original author and source are credited.

\begin{abstract}
Cancer screening is an approach to reduce cancer-related morbidity and mortality rates. Among those screening tests that have been shown to lead to reduction in cancer deaths 2 tissue biomarkers, PSA and CA-125 have shown to contribute to clinical utility. No tumor marker has however been identified to date that is sufficiently sensitive or specific to be used on its own to screen for cancer. Screening for circulation tumor cells as a new biomarker in the blood therefore seems like an attractive possibility. Cancer is known to spread circulating tumor cells before symptoms occur. Therefore, we have screened 1,585 asymptomatic individuals of age 20 to 80 with no known cancer risk factors. 27 individuals showed one or more circulating tumor cell in a $7.5 \mathrm{ml}$ blood sample, which constitutes a positive circulating tumor cell test, based on the iCellate IsoPic ${ }^{\mathrm{TM}}$ laboratory test. That number compares favourably with the cancer incidence of 157.1 per 100,000 people per year in Peru, given that the screening results would be expected to correspond to an accumulated incidence of up to 12 years. The present findings therefore identify circulating tumor cells as a promising new cancer screening test.
\end{abstract}

Keywords: Clinical; CTC isolation; Screening; Tumor cells; Cancer; Technique

\section{Introduction}

Epithelial cancers spread by inappropriately invading the basement membrane of extracellular matrix fibers, a key microscopic observation by clinical pathologists that forms part of the diagnosis of cancer. The invading cells may invade locally or may enter the lymph or blood vessels [1]. The result is local or systemic spread of the cancer cells that may result in metastases. Clinical observation and experimental testing demonstrate that the spread can occur early in the disease progression, well before the primary tumor would give symptoms or be visible by medical imaging $[2,3]$. Screening for CTCs in the blood is therefore an inherently promising approach to early detection of cancer.

The first generation of CTC isolation instrumentation, which used a cell surface biomarker as an immune-affinity tag for cancer cell detection [4], has not fulfilled the promise of early detection of cancer $[5,6]$. This may be related to the limitation of the cell surface marker used, EpCAM, since it may be lost during disease progression or depending on the state of the cells. The understanding is that a more universal, marker independent $2^{\text {nd }}$ generation, approach to CTC isolation is required [5]. One such $2^{\text {nd }}$ generation CTC isolation instrument is the iCellate's IsoPic ${ }^{\mathrm{TM}}$. In addition it has previously been demonstrated that an IsoPic ${ }^{\mathrm{TM}}$ prototype could isolate CTCs from a patient with peritoneal carcinomatosis [7]. The isolated cells could also be demonstrated to be genetically abnormal, consistent with being cancerous (Castro and Ericsson, unpublished data). The iCellate
IsoPic ${ }^{\mathrm{TM}}$ would therefore seem to be a promising test to screen asymptomatic individuals for suspicious cells that can then be tested further, aiming for the screening leading to the early detection of cancer.

In the present paper we report the results of screening 1,585 subjectively healthy individuals, with no previous cancer diagnosis. We find 27 individuals carrying cells in their blood that satisfy the established criteria for CTCs. The iCellate IsoPic ${ }^{\mathrm{TM}}$ therefore seems to provide a promising diagnostic test for the early detection of cancer.

\section{Materials and Methods}

ICELLATE1 is all-comers, single center trial including individuals in the age of 20-80 years not belonging to any known risk group. Any individual with a current diagnosis or history of any types of tumor was excluded.

All experimental protocols were approved by the Instituto Nacionalde Salud, Comité de Investigación, Lima, Peru. The methods were carried out in accordance with the relevant guidelines and regulations. Informed consent was obtained from all subjects.

\section{- Blood draw}

The subjects provided $7.5 \mathrm{ml}$ of blood for CTC analysis.

\section{- CTC isolation}

CTC isolation was carried out within $24 \mathrm{~h}$ after the blood draw with iCellate's isolation technology according to the IsoPic ${ }^{\mathrm{mx}}$ operators' manual. The IsoPic ${ }^{\mathrm{ma}}$ system is intended to, from a blood sample, 
identify, concentrate and purify CTC to make them available as a sample to support the enumeration and analyses to support diagnosis of metastatic tumors and those non-metastatic tumors that may release circulating tumor cells.

\section{- Cell staining}

DAPI (4', 6-Diamino-2-phenylindole dihydrochloride, Cat. no. D9542-5mg, Sigma-Aldrich)

Anti-Pan cytokeratin (AE1/AE3)-efluor570, (Cat. no. 41-9003-82, eBioscience)

\section{Anti-Human CD45 (2D1)-FITC (Cat. no. 345808, BD)}

\section{- Microscopic evaluation}

Microscopic evaluation was carried out by two independent experienced operators.

\section{Results}

1,585 subjectively healthy people, with no previous history of cancer, were screened for the presence of any cells in a $7.5 \mathrm{ml}$ blood sample that comply with the established definition of a CTC. Those cells were counted (Figure 1). 1,558 subjects were negative for any such cells. 27 samples were positive for CTCs (Table 1). Typical images of immunofluorescence staining of circulating tumor cells can be found [10]. The numbers of CTCs varied from 1 to 6 . The relative numbers of subjectively healthy study persons with CTCs among those with no known cancer risk factors was found to be $1,7 \%$, which is notably lower than the 3,2\% found among the like-wise subjectively healthy, but at-risk, study persons [10], i.e., exposure to one or more cancer risk factors increase the likelihood to also have CTCs. The results suggest that screening for CTCs is already a valuable screening tool, whose merits would be further enhanced by further clinical follow up and by molecular analyses of the isolated cells.

\section{Discussion}

The present study found that cells meeting the established criteria for CTCs can indeed be detected among asymptomatic individuals with no known increased risk of cancer, suggesting those cells may be a promising new sample type for cancer health screening and early detection. These findings are in line with the clinical information that primary tumor spread metastases by way of the blood years before they give symptoms and can be diagnosed by medical imaging, but was by no means self-evident [2]. The, since the year 2004, FDA approved Cell Search technique (for late stage breast, prostate or colorectal cancer) based on immune affinity isolation of EpCAM cell surface marker expressing cells in blood samples has not been able to meet the expectations of establishing a screening test for spreading cancer [4-6]. The discrepancy may be due to the Cell Search method relies on a cell surface marker that may not be present on all cancer cells, making the IsoPic ${ }^{\mathrm{TM}}$ both more sensitive and more representative of the entire panorama of cancer cells.

The age-standardized cancer rate, incidence per 100,000 people per year is 157.1 in Peru, or about one-tenth of the CTC incidence found in the present study. We think there are two reasonable explanations for the discrepancy between the clinical incidence of cancer and the incidence of CTCs: 1) since the spread of a tumor may occur up to 12 years [2] before onset of symptoms, and given that the tumor spreads by means of CTCs, we think up to 12 multiples of the yearly clinical incidence may be reasonable for the CTCs, based on the assumption that any cancer developing clinically one year later, two years later, and so on up to 12 years later would be discovered at any one given time by the CTC screen, but before symptom debut. 2) There may exist a spontaneous regression such that some individuals would be able to eliminate any CTCs so they do not progress to clinical cancer [8].

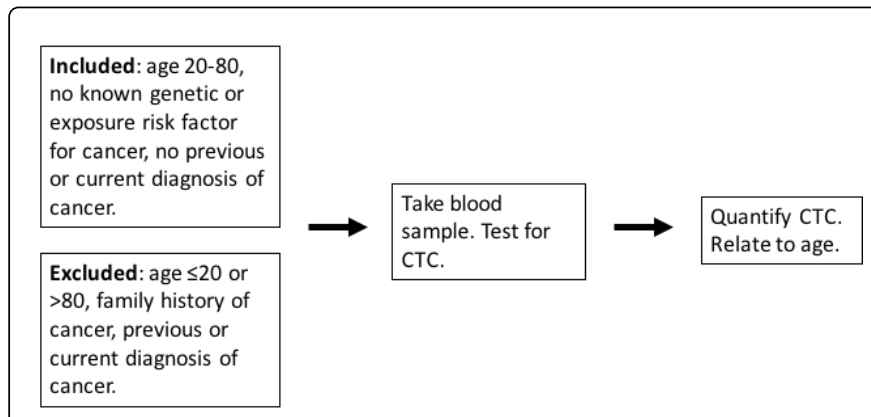

Figure 1: Schematic representation of this study including inclusion/exclusion criteria.

\begin{tabular}{|l|l|l|l|l|l|l|l|l|}
\hline Age & Total \# pat & $\mathbf{2}$ & $\mathbf{3}$ & $\mathbf{4}$ & $\mathbf{5}$ & $\mathbf{6}$ & $\begin{array}{l}\text { \# CTC pos } \\
\text { pat }\end{array}$ & $\%$ \\
\hline $20-24$ & 215 & 1 & - & - & - & - & 1 & 0.5 \\
\hline $25-29$ & 190 & 3 & 2 & 1 & 1 & - & 7 & 3.7 \\
\hline $30-34$ & 149 & - & - & 1 & - & - & 1 & 0.7 \\
\hline $35-39$ & 140 & 2 & - & 1 & - & - & 3 & 2.1 \\
\hline $40-44$ & 148 & - & - & 1 & - & - & 1 & 0.7 \\
\hline $45-49$ & 238 & 3 & 2 & - & - & - & 5 & 2.1 \\
\hline $50-54$ & 150 & 6 & - & - & 1 & - & 7 & 4,7 \\
\hline $55-59$ & 129 & 1 & - & - & - & - & 1 & 0.8 \\
\hline $60-64$ & 98 & - & - & - & - & 1 & 1 & 1 \\
\hline- & 1585 & 0 & 0 & 0 & 0 & - & 27 & 1.7 \\
\hline
\end{tabular}

Table 1: Numbers of circulating tumor cells relative their age, for individual subjects. 1 to 6 circulating tumor cells per $7.5 \mathrm{ml}$ blood sample in 27 individuals out of the 1,585 screened.

The first generation CellSearch technique showed that 8 samples contained CTCs observable by that technology in the 145 healthy subjects tested (5.5\%). Similarly, 14 of the 199 women with benign breast diseases had CTCs (7.5\%) those findings were used to adjust the base line of what was considered a positive CTC test [9]. A recent screening study of at risk individuals showed a higher CTC incidence of $3.2 \%$ compared to the present standard risk cohort that shows a lower $1.7 \%$ CTC incidence [10]. Thus, we think the CTC findings in this study entirely align with reasonable assumptions of disease mechanism, with the cancer incidence and with findings previously reported in the scientific literature. To our knowledge no other publication exists of a large cohort of study persons, whether without known risk factors for cancer or with increased risk for cancer, having been screened for CTCs. Future clinical follow-ups, using established criteria for early cancer detection [11], and more detailed analyses of the CTCs will serve to further clarify the relationship between CTC and cancer incidence. 
The established criteria for identification of CTCs as such, DAPI positivity, cytokeratin positivity and CD45 negativity demonstrate only that the isolated cells show traces of being epithelial and not of being an immune cell, but do not in and of themselves demonstrate the cell is a cancer cell [12]. Epithelial cells should however not be present in the circulation. Their very existence is therefore a warning sign and a suspicious finding that should trigger a clinical follow up, since no CTCs should be present in circulation. Future clinical trials will establish the opportunities and limitations of this new CTC detection and quantification. In the meantime, since a screening test is not intended to be diagnostic on its own, individuals with suspicious findings must be referred to a physician for diagnosis and any consequent treatment [13]. Established criteria for the early detection of a variety of cancers exist [11]. Following up on a positive CTC test with established cancer early detection may be medically and health economically advantageous.

Previous experimental studies of cancer have shown that only genetically abnormal cells can seed metastases, not similar cells with functionally normal genomes [14]. To bring cell based diagnosis of cancer to its logical conclusion therefore requires that the isolated cells are also subjected to either functional tests for the hallmarks of cancer or are genetically sequenced, followed by the relevant bioinformatics analysis, to establish their cancerous nature $[15,16]$. Such tests, in conjunction with the isolation of the inappropriate cells would be expected to enhance the clinical relevance of the CTC detection and quantification. It should therefore be clear that to go beyond a suspicious finding and towards establishing that the cells are actually cancerous, even if at a very early stage, will require genetic analyses of the cells. For those cases, the present sample type would provide an ideal sample.

It will also be of interest to compare the genetic analyses of isolated CTCs to those of free circulating tumor DNA, ctDNA, to establish the merits of each sample type. The ctDNA sample is easily available, but is degraded, diluted, admixed with normal DNA and has lost its cellular context, but may still provide a useful alternative when those negative factors are of lesser importance. Since the CTCs are the actual vehicles of metastatic spread and provide a highly concentrated and pure sample in its cellular context, it would be expected that CTCs would remain the "gold standard" of blood based cancer testing.

Summary: CTCs can be detected in a-symptomatic individuals. A positive CTC-screening test is a suspicious finding that should be followed up clinically. The value of the test will be further enhanced following clinical trials and by adding new molecular tests of the isolated circulating tumor cells.

\section{Acknowledgement}

The authors are pleased to acknowledge the receipt of a EUREKA and European Community grant from the Eurostars program, grant number E!8445 - CellCleanUpSystems to Christer Ericsson.

\section{Author Contributions Statement}

L.S. contacted the patients and oversaw the blood sampling and clinical record keeping, P.H.A.B evaluated the cells, MTN oversaw the circulating tumor cell isolation and associated record keeping and the transfer of the processed samples for laboratory analyses ML, TC and HRS performed the laboratory analyses, cell staining, microscopic analyses and associated record keeping JC and CE conceived of the experiments, $\mathrm{CE}$ analyzed the results and authored the manuscript. All authors reviewed the manuscript.

\section{Additional Information}

\section{Competing financial interests}

Dr. Castro, Dr. Luis Sanchez, Dr. Pierino H. Alvarez Bedoya, Dr. Maria Teresa Nunez, Dr. Ming Lu, Tomas Castro and Dr. Hamid Reza Sharifi declare no potential conflict of interest. Dr. Ericsson owns stock in iCellate Medical and is employed as its vice president for research and development (VP R\&D).

\section{References}

1. Fujii T, Sutoh T, Morita H, Yajima R, Yamaguchi S, et al. (2014) Vascular invasion, but not lymphatic invasion, of the primary tumor is a strong prognostic factor in patients with colorectal cancer. Anticancer Res 34: 3147-3151.

2. Friberg S, Mattson S (1997) On the growth rates of human malignant tumors: implications for medical decision making. J Surg Oncol 65: 284-297.

3. Husemann Y, Jochen BG, Falk S, Piero M, Manfred M, et al. (2008) Systemic spread is an early step in breast cancer. Cancer cell 13: 58-68.

4. Cristofanilli M, Budd GT, Ellis MJ, Stopeck A, Matera J, et al. (2004) Circulating tumor cells, disease progression, and survival in metastatic breast cancer. N Engl J Med 351: 781-791.

5. Paxton A (2014) Unraveling metastasis with circulating tumor cells. CAP Today.

6. Swennenhuis JF, van Dalum G, Zeune LL, Terstappen LW (2016) Improving the Cell Search (R) system. Expert Rev Mol Diagn 16: 1291-1305.

7. Castro J, Ericsson C, Cashin P, Mahteme H (2012) Preliminary finding: detection of circulating cancer cells in blood from a patient with peritoneal carcinomatosis treated with cytoreductive surgery and intraperitoneal chemotherapy. Surgery Curr Res 2: 2161-1076.

8. Papac RJ (1998) Spontaneous regression of cancer: possible mechanisms. In vivo (Athens, Greece) 12: 571-578.

9. Allard WJ, Matera J, Miller MC, Repollet M, Connelly MC, et al. (2004) Tumor cells circulate in the peripheral blood of all major carcinomas but not in healthy subjects or patients with non-malignant diseases. Clin Cancer Res 10: 6897-6904.

10. Castro J, Sanchez L, Nunez MT, Lu M, Castro T, et al. (2018) Screening circulating tumor cells as a non-invasive cancer test in 3388 individuals from high-risk groups (ICELLATE2). Dis Markers 2018.

11. Smith RA, Andrews K, Brooks D, De Santis CE, Fedewa SA, et al. (2016) Cancer screening in the United States, 2016: A review of current American Cancer Society guidelines and current issues in cancer screening. CA Cancer J Clin 66: 96-114.

12. Racila E, David E, Arthur JW, Chandra R, John MC, et al. (1998) Detection and characterization of carcinoma cells in the blood. Proc Natl Acad Sci USA 95: 4589-4594.

13. Wilson JMG, Jungner G (1968) Principles and practice of screening for disease. Vol Public Health Papers 34.

14. Podsypanina K, Du YCN, Jechlinger M, Beverly LJ, Hambardzumyan D, et al. (2008). Seeding and propagation of untransformed mouse mammary cells in the lung. Science 321: 1841-1844.

15. Hanahan D, Weinberg RA (2011) Hallmarks of cancer: the next generation. Cell 144: 646-674.

16. Hanahan D, Weinberg RA (2000) The hallmarks of cancer. Cell 100: 57-70. 